La cuisine coréenne comme représentation de la communauté nationale : analyse des rapports entre communauté et cuisine dans les films «Sikgaek » et « Sikgaek 2 »

\title{
Aurelien PASQUIER
}

\section{OpenEdition}

\section{Journals}

Édition électronique

URL : http://journals.openedition.org/transtexts/631

DOI : 10.4000/transtexts. 631

ISSN : 2105-2549

Éditeur

Gregory B. Lee

Référence électronique

Aurelien PASQUIER, « La cuisine coréenne comme représentation de la communauté nationale

analyse des rapports entre communauté et cuisine dans les films « Sikgaek » et « Sikgaek 2 » »,

Transtext(e)s Transcultures 跨文本跨文化 [En ligne], 10 | 2015, mis en ligne le 08 août 2016, consulté le 20 avril 2019. URL : http://journals.openedition.org/transtexts/631 ; DOI : 10.4000/transtexts.631

Ce document a été généré automatiquement le 20 avril 2019.

(c) Tous droits réservés 


\title{
La cuisine coréenne comme représentation de la communauté nationale : analyse des rapports entre communauté et cuisine dans les films «Sikgaek » et «Sikgaek 2 »
}

\author{
Aurelien PASQUIER
}

\section{De la libéralisation à la protection de la culture nationale}

1 Après la colonisation japonaise, la Corée du Sud fut un des pays les plus pauvres dès son établissement en 1948. Jusqu'au début des années 1960 le pays vécut principalement de l'aide humanitaire des États-Unis. À partir des années 1960, Park Chung-hee mena la Corée du Sud sur le chemin de la croissance économique. Cependant, sans parler de l'impossibilité d'opposition politique, les droits des travailleurs et les salaires furent sacrifiés sur l'autel de la croissance économique. Avec la fin des dictatures militaires et la démocratisation, la Corée du Sud, sous la pression des États-Unis, s'engagea dans une politique de libéralisation de son économie à partir du milieu des années 1980. Cette période de dérégulations présenta un danger pour les industries nationales qui perdirent des parts de marché. L'arrivée sur les étalages de nombreux produits étrangers et l'occidentalisation de la société sud-coréenne devinrent une source d'inquiétude et posèrent la question de l'identité nationale. Durant sa présidence, Kim Young-sam entreprit de faire évoluer l'économie sud-coréenne dans la mondialisation. Non plus de la subir, mais de s'inscrire en son sein afin que l'économie sud-coréenne ne soit plus au service de la mondialisation, mais que la mondialisation soit au service de l'économie sudcoréenne. C'est pour cette raison que Kim Young-sam lança sa politique de mondialisation segyehwa 세계화. La mondialisation se devait d'être une politique de "coréanisation " 
hangukhwa한국화. La mondialisation et le concept d'économie nationale forte ou d'Étatnation, si elle semblent contradictoires, entretiennent en réalité une relation plus complexe qu'un simple antagonisme. Le néo-libéralisme apparu depuis plusieurs décennies n'a pas réussi à la destruction « prophétisée » par certains chercheurs de l'Étatnation. La mondialisation représenta, et est toujours, un moyen pour la Corée du Sud de se développer économiquement, mais également de renforcer l'identité nationale par une volonté des différents gouvernements depuis les années 1990 de jouer leur rôle de régulateur de l'économie, au travers des politiques de soutien et de défense des industries du pays.

\section{Émergence d'une nouvelle classe économique et la philosophie sinto buli}

2 Maria Osetrova explique le développement des études anthropologiques sur la nourriture en Corée du Sud à partir des années 1990 par l'apparition d'une génération née dans les années 1970 n'ayant pas connu de famine et jouissant de moyens financiers leur permettant de consommer selon leurs envies. ${ }^{1}$ Le gouvernement sud-coréen décida d'organiser des festivals afin de défendre la culture traditionnelle et populaire sudcoréenne. L'aisance économique des nouvelles classes moyennes popularisa le well-being, mais le plus grand symbole de l'essor de cet intérêt croissant pour la gastronomie et de la volonté des dirigeants sud-coréens est la politique sinto buli 신토불이 (身土不二). Shin Gi-Wook décrit le développement et l'importance atteinte par cette philosophie dans la Corée du Sud actuelle ainsi :

3 Reflecting such state policy, sint'o puli (body and soil as the same) discourse has become popular in the 1990s. As the term implie, sint'o puli claims that if a person has Korean blood, he or she is Korean, regardless of class background or place of residence. Therefore, it can be claimed that Koreans remain Korean because they share the same blood. Neither globalization not any other social change can alter this fact. Moreover, the ideology of sint'o puli has not remained at the level of discourse. It has been transformed into the commercialization of the Korean culture and heritage. Food, drink, and either everyday commodities that incorporate traditional «Korean » elements have become popular products in the Korean market. ${ }^{2}$

4 C'est également à partir de cette même période que la culture culinaire devint de plus en plus une source de fierté pour les Sud-Coréens. ${ }^{3}$ Une des raisons de la revalorisation de la gastronomie sud-coréenne fut celle de ses bienfaits pour la santé et notamment ses qualités dans la prévention des cancers. Le kimchi était une source de honte jusqu'au début des années 1960 en Corée du Sud. ${ }^{4}$ Cet embarras pour le kimchi venait de l'époque coloniale durant laquelle il était source de discrimination à cause de sa forte odeur de fermentation. ${ }^{5}$ La prise de conscience des bienfaits supposés du kimchi et l'augmentation de ses exportations firent qu'il obtint un nouveau statut au sein de la société sudcoréenne :

[...] kimchi is becoming the symbol of cultural distinctiveness to be defended from within as well as from without. ${ }^{6}$ 
Ce fut également pour ces raisons que la perception du kimchi évolua au Japon à partir du milieu des années 1990. En outre, après la libéralisation de la culture japonaise en 1998, une part des médias japonais présenta le kimchi comme étant bon pour la santé.

\section{Défendre le kimchi contre le kimuchi}

7 Une des revendications du gouvernement sud-coréen afin de défendre les entreprises sud-coréennes agro-alimentaires et la gastronomie sur le marché mondial concerna le kimchi. En 1988, à l'occasion des Jeux Olympiques organisés en Corée du Sud, le gouvernement décida de choisir le kimchi et le bulgogi comme plats représentatifs de la gastronomie nationale. L'obtention du statut de plat national pour le kimchi marque une confiance grandissante des Sud-Coréens dans leur propre culture. ${ }^{7}$ En 1996, le gouvernement sud-coréen protesta auprès de la commission du codex Alimentarius contre les produits japonais, accusés de dévoyer l'authenticité du vrai kimchi, et demanda que leurs produits aient une autre appellation ${ }^{8}$. Le gouvernement sud-coréen réussit à faire adopter des normes en 2001 pour tout produit portant l'appellation de kimchi :9

prepared from varieties of Chinese cabbage, Brassica pekinensis Rupr.; such Chinese cabbages shall be free from significant defects, and trimmed to remove inedible parts, salted, washed with fresh water, and drained to remove excess water; they may or may not bec ut into suitable sized pieces/parts ;

processed with seasoning mixture mainly consisting of red pepper (Capsicum annum L.) powder, garlic ginger, edible Allium varieties other than garlic, and radish. These ingredients may be chopped, sliced and broken into pieces; and

fermented before or after being packaged into appropriate containers to ensure the proper ripening and preservation of the product by lactic acid production at low temperatures.

Le Ministère de l'agriculture et des forêts sud-coréen décrit ainsi la différence entre le kimchi (prononcé kim-chi), et le kimchi japonais (prononcé ki-mu-chi) :

한국의 김치는 절임배추에 여러가지 양념류를 혼합해 젖산 발효에 의해 만든 식 품인데 비해 일본의 기무치는 단순한 겉절이 식품에 불과하다. ${ }^{10}$

Alors que le kimchi sud-coréen résulte de la fermentation de choux chinois saumurés et mélangés avec diverses épices, le kimuchi japonais n'est que du geotjeol'i (kimchi non fermenté).

On peut noter que le choix du terme kimuchi pour parler des produits japonais permet de les mettre en opposition au kimchi sud-coréen soulignant que la différence entre les deux dépasse le cadre linguistique. Toutefois, le kimchi tel que défini dans le codex Alimentarius est en fait lui-même un mets relativement récent dans l'histoire coréenne. Bien que les origines du kimchi fassent débat parmi les spécialistes, l'introduction de la poudre de piment date d'il y a un peu moins de trois siècles. ${ }^{11}$ Cependant, aujourd'hui le kimchi est un des plats les plus, si ce n'est le plus, représentatif de la cuisine sud-coréenne dans la péninsule et à l'étranger. Sa nouveauté dans la gastronomie coréenne n'atténue en rien son pouvoir symbolique, comme le prouve l'exemple du thé en Angleterre. Le thé est aujourd'hui considéré comme la boisson par excellence des Anglais en Occident, mais l'apparition du thé dans les îles anglo-saxonnes remonte seulement à l'impérialisme britannique.

Toujours dans l'objectif de renforcer l'image de la culture culinaire, et de ce fait l'identité nationale, le gouvernement chercha à faire inscrire au patrimoine immatériel de l'UNESCO le kimchi et le kimjang. ${ }^{12}$ Ces efforts furent récompensés en décembre 2013 avec 
l'inscription au patrimoine immatériel de l'UNESCO du kimchi et du kimjang. Cette reconnaissance par une institution internationale fut également une victoire symbolique sur le Japon. Dans son enquête sur la mondialisation, Shin Gi-wook pointe l'importance d'internet dans la construction du nationalisme sud-coréen depuis le milieu des années $1990 .{ }^{13}$ Cette volonté d'utiliser internet comme moyen de faire connaître et reconnaître sa gastronomie à l'international par le gouvernement est manifeste avec la création du site Hansik Globalization Project traduit en plusieurs langues. Ce site est accompagné de comptes sur tous les réseaux sociaux populaires actuels, Twitter, Facebook et Youtube. Une section sur le site est même réservée à la «nécessité de la mondialisation de la gastronomie sud-coréenne » hansik segyehwa pilyoseong 한식 세계화 필요성.

한식의 우수성을 바탕으로 한식을 발전시키고 한식문화의 국내외 확산을 통해 농 림축산식품산업, 외식산업, 문화관광산업 등 관련산업을 발전시키고 대한민국 이 미지를 향상시키고자 하는 것이 한식 세계화 입니다. ${ }^{14}$

La mondialisation de la cuisine sud-coréenne (hanshik) a pour but d'améliorer l'image de la Corée du Sud et de développer les différentes industries concernées (industries culturelles, restauration, industries du Ministère de l'agriculture et de l'alimentation, etc.) via la diffusion de la culture gastronomique sud-coréenne et le développement de la cuisine sud-coréenne en se basant sur son excellence.

\section{La gastronomie dans la culture populaire}

11 Alors que les dictatures militaires subventionnaient presque exclusivement la culture traditionnelle, les gouvernements démocratiques prenant conscience de l'importance de la culture populaire dans l'économie capitaliste contemporaine, se mirent à soutenir les industries culturelles, vues désormais comme une source de richesse nationale ainsi qu'un moyen de populariser la culture sud-coréenne. Même s'il critiqua son prédécesseur pour n'avoir pas prévu la crise, Kim Dae-jung continua une politique semblable à Kim Young-sam. Cette continuité des politiques fut observable notamment dans l'industrie cinématographique. La période de libéralisation lui fut presque fatale. Elle continua de perdre des parts de marché pour atteindre son indice le plus faible de son histoire en 1993..$^{15}$ Même si une grande partie des politiques pour soutenir l'industrie cinématographique décidées par Kim Young-sam ne prirent effet que lors de la mandature de son successeur, l'industrie cinématographique actuelle acquit ses bases à ce moment-là avec un marché dominé par les chaebol. Cependant, les chaebol, principalement Samsung, Hyundai et Daewoo, décidèrent après la crise financière asiatique de 1997 de se recentrer sur leurs activités principales et de se séparer de leurs filiales déficitaires dont faisaient partie leurs sociétés de production de cinéma. Après 1997, de nouveaux acteurs apparurent sur le marché national comme CJ Entertainment et Lotte, permettant ainsi de repasser la barre symbolique des $50 \%$ de part de marché en $2001 .^{16}$

\section{"Sikgaek », vitrine de la gastronomie de la cuisine sud-coréenne}

Ce renouveau de la cuisine traditionnelle fut également visible dans les médias. Par un effet de résonance, cette reconsidération de la cuisine sud-coréenne dans les médias permit de renforcer la popularité grandissante de la gastronomie nationale. Le phénomène le plus représentatif de la popularité acquise par la culture coréenne à l'étranger, bien que cela concerne majoritairement l'Asie, est la « vague coréenne » Hallyu 
한류. Parmi les différentes séries télévisées exportées devenues très populaires, Dae Janggeum 대장금, diffusée en Corée du Sud en 2003 sur la chaîne MBC, fut une des premières à faire connaître la culture coréenne à l'étranger. Cette série longue de 52 épisodes devint une vitrine de la gastronomie sud-coréenne. Les plats visibles dans cette série télévisée étaient préparés par des personnes de l'Institut de la cuisine royale coréenne ( www.food.co.kr), parmi lesquelles Hwang Hye-song, chercheuse qui participa grandement à la popularisation de la cuisine royale coréenne à travers les médias. ${ }^{17}$ Elle participa également au film "Sikgaek " $^{18}$, aussi connu sous le nom «Le Grand Chef » (2007).

13 «Sikgaek » est basé sur le manwha (bande dessinée sud-coréenne) éponyme publié dans le journal Dong'a ilbo du 2 septembre 2002 au 17 décembre 2008. Il servit également de base à un spin-off, n'ayant que le personnage principal Seong-chan de commun avec l'œuvre originale, en 2009, «Sikgaek : la guerre du kimchi » 식객 : 김치전쟁. Alors que le premier film connut une popularité notoire dans les salles avec plus de trois millions de spectateurs, le second n'attira guère les foules, avec seulement 462000 personnes $^{19}$, bien qu'ils aient été projetés sur un nombre similaire d'écrans ${ }^{20}$ et que le deuxième opus ait profité également d'une publicité supplémentaire grâce aux deux acteurs principaux Kim Jeong-eun et Jin-Goo nommés ambassadeurs du kimchi à Gwangju en $2009^{21}$. La popularité du premier film et du manwha donna, semble-t-il, confiance à des producteurs avec la création et la diffusion d'une série télévisée «Sikgaek» en 2008. Les deux films furent distribués par deux des quatre plus grands chaebol présents dans l'industrie cinématographique actuellement. Le premier par CJ Entertainment et le deuxième par Lotte Entertainment (également connu comme Lotte Cinema). Ces deux sociétés sont respectivement des filiales de CJ Group et Lotte. Il n'est sûrement pas sans intérêt de souligner que ces deux groupes, à l'instar de beaucoup d'autres, produisent également des produits alimentaires dont du kimchi. Toutefois, bien que les deux films aient été réalisés par des sociétés différentes et des réalisateurs différents, on pourra noter que le scénariste Shin Dong-ik participa à l'écriture des deux films.

Dans le premier film, Seong-chan et Bong-ju s'opposent lors d'un grand concours national. Les deux protagonistes se connaissent depuis longtemps pour être allés étudier dans le même institut de cuisine royale. Cependant, tout les oppose. Bong-ju, grand favori, finit par perdre lors de l'ultime épreuve face à Seong-chan à cause de son manque de respect des produits. De plus, alors que Seong-chan s'occupe de son grand-père sénile, qui le guidera malgré son décès vers la victoire, Bong-ju est quelqu'un de vil et qui n'hésite pas à tricher en mettant la vie de personnes en danger pour gagner. Dans le spinoff, Seong-chan affronte sa demi-sœur Jang-eun dans un concours national qui doit permettre de déterminer le meilleur cuisinier de kimchi de la Corée du Sud.

La construction de l'histoire dans les deux films est similaire. L'intrigue oppose deux personnages liés par leur passé autour d'une compétition de cuisine. De plus, nous pouvons remarquer que l'affrontement entre Seong-chan et son adversaire ne se limite pas au concours, mais que leur mode de vie lui-même les oppose totalement. Dans le premier film, alors que Bong-jun possède une chaîne de restaurant, est interviewé à la télévision et fait des affaires à l'étranger, Seong-chan vend des produits à l'arrière de sa camionnette et est proche des gens. De même, alors que le Seong-chan du second opus vend lui aussi des légumes et des fruits dans des marchés à l'arrière de sa camionnette, sa sœur est devenue une chef réputée au Japon où elle a habité pendant dix ans. Après son 
retour au pays, elle est invitée sur des plateaux de télévision afin de parler des raisons de son retour, de ses envies et de son futur.

En outre, on soulignera que dans chacun des films la compétition oppose les deux protagonistes sur deux points, à savoir : premièrement, déterminer qui est le meilleur des deux afin de remporter la victoire, mais également devenir le représentant de la Corée du Sud ; deuxièmement, définir celui qui obtiendra la souveraineté du couteau du dernier cuisinier royal dans « Sikgaek », et du restaurant de leur mère dans « Sikgaek 2 ».

\section{Le spectacle de la gastronomie nationale}

17 Même s'il est possible de dégager d'autres problématiques au sein de ces deux films telles que la famille, la répétition de l'histoire, ou bien encore la traîtrise, nous nous limiterons dans cette étude à celle du nationalisme et plus particulièrement à la thématique de la construction de l'identité nationale sud-coréenne contemporaine en relation avec la culture gastronomique nationale.

Dans un article du journal Jung'ang ilbo, le critique de cinéma Cho Jae-eun écrit que le deuxième opus force la reconnaissance du traditionnel sur le moderne ${ }^{22}$. Toutefois, comme nous venons de le voir, les deux films suivent une même structure avec le personnage principal, Seong-chan, qui représente la tradition, face à son adversaire qui incarne la modernité. Qu'il s'agisse de Bong-jun ou de Jang-eun, les deux protagonistes donnent l'impression de s'être éloignés de leurs racines. Bong-jun est en fait celui qui a piégé Seong-chan lorsqu'ils étaient tous les deux étudiants dans une école de cuisine royale en empoisonnant le plat de ce dernier par jalousie, ne pouvant rivaliser avec le talent de cuisinier de Seong-chan. Ceci lui permit de diriger "Unmajeong ", restaurant prestigieux, et de devenir un homme d'affaire très riche méprisant toute chose non dispendieuse. Jang-eun, quant à elle, se rendit au Japon afin de s'éloigner de sa mère dont elle avait honte.

La principale différence entre les deux personnages opposés à Seong-chan est que Jangeun revient vers ses racines à travers le concours, chaque épreuve lui faisant re-découvrir le prestige de la cuisine et des produits sud-coréens. Bong-jun, lui, s'entête dans ses choix et finit par tout perdre.

Dans le premier « Sikgaek », le plat proposé par Seong-chan est un yukkejang. Bien que les juges le raillent car c'est un plat du peuple, il s'agira en réalité bien du dernier plat préparé par le chef royal coréen. Cependant, cette opposition entre la modernité et la tradition n'est pas qu'une simple dichotomie. De même que la tradition ne signifie pas le refus de la modernité, la modernité ne signifie pas le refus de la tradition. La dernière épreuve du concours de chaque film montre la volonté du gouvernement d'inscrire la tradition dans la modernité. Dans le cas du second film, le but est de réaliser un kimchi qui puisse plaire au monde entier. Les deux personnages principaux font, contre toute attente, le même kimchi. Ils estimèrent en effet que le meilleur kimchi possible était celui de leur mère ommaui mat 엄마의 맛. Ce qui permet de remporter la victoire dans le second film est donc un kimchi traditionnel. Le kimchi, qui est censé représenter la Corée du Sud mondialement, n'est pas un kimchi adapté au goût des étrangers, comme si tous les étrangers avaient un même goût et de surcroît le respect de la tradition. La cuisine sudcoréenne ne doit alors pas s'adapter, ni dans le goût et ni dans le processus de fabrication, 
au reste du monde, mais la mondialisation sert à renforcer le prestige de la gastronomie sud-coréenne et sa découverte à travers le monde.

Alors que dans le premier film le concours sert à retrouver l'identité coréenne perdue dans la colonisation et par allégorie dans la mondialisation, le deuxième film sert à montrer le chemin afin de "globaliser » la gastronomie (et donc la culture) sud-coréenne dans le monde actuel. Dans les deux cas, il s'agit de retrouver les racines coréennes dans la société mondialisée actuelle.

Claude Fischer explique que « la nourriture est centrale à notre sens de l'identité $»{ }^{23} \mathrm{La}$ consommation d'aliment peut ainsi être un moyen de marquer son identité culturelle. Le fait de revaloriser le prestige de la gastronomie sud-coréenne devint un moyen de consolider l'identité nationale. Claude Fischer ajoute également :

Incorporation is also the basis of collective identity and, by the same token, of otherness. Food and cuisine are a quite central component of the sens of collective belonging. ${ }^{24}$

La défense du kimchi dans le cas de la Corée du Sud représente d'un côté la protection et un renforcement de la construction de l'identité nationale, mais permet également de créer une différence avec les autres nations. Avec la démocratisation de la Corée du Sud et l'apparition de la "génération 386 ", la Corée du Nord n'apparaissait alors plus comme un ennemi mais comme un frère. À partir de la fin des années 1980, le Japon devint « l'ennemi national ». Kimura Kan justifie ce changement par la nécessité du nationalisme sud-coréen d'avoir un "ennemi fictif » kasō teki sagashi仮想敵探し. ${ }^{25}$ Toutefois, comme l'écrit Kozakai Toshiaki, tout groupe se forme en opposition à un autre groupe. Autrement dit, pour qu'un « Nous » existe il faut un «Eux ». ${ }^{26}$ Le besoin d'un «Autre » pour qu'un «Soi » existe n'est donc en rien une spécificité du nationalisme sud-coréen.

Le point de départ des deux films est lié au Japon. Dans le premier, après un rapide retour sur les faits qui se sont passés entre les deux protagonistes cinq années auparavant, le film enchaîne sur une conférence de presse où le fils d'un ancien gouverneur général de Corée est venu rapporter le couteau utilisé par le dernier chef cuisinier royal. Ce dernier avait préféré se couper la main plutôt que de préparer à manger pour les colonisateurs nippons. Alors que le premier film aborde la gastronomie par le passé colonial, le deuxième fait référence aux conflits territoriaux récents qui opposent les deux pays. Le titre du second opus lui-même est sans équivoque, «Sikgaek 2 : la guerre du kimchi » 식 객2: 김치전쟁. La «guerre du kimchi » fait naturellement référence à la compétition entre les deux personnages principaux, mais induit plus encore que la guerre pour le kimchi est une guerre contre le Japon. Cette lutte pour la souveraineté fait directement référence aux mots du Premier Ministre japonais au début du film. Lors d'un dîner officiel avec son homologue sud-coréen, celui-ci déclare :

今、召し上がっているのは焼肉とキムチです。我が国の代表的な料理です。

Vous êtes en train de manger du kimuchi et du yakiniku. Des plats représentatifs de

la cuisine de notre pays.

Comme nous l'avons vu précédemment, le kimchi fut une source de conflit au milieu des années 1990 entre les deux pays. Cependant, si l'on considère la gastronomie comme un élément de la construction de l'identité culturelle, l'appropriation d'un plat par un autre pays peut être considérée comme une atteinte à sa souveraineté. Hormis les problèmes liés à l'histoire coloniale, une des questions pendantes entre les deux pays est celle de la souveraineté de Dokdo/Takeshima. ${ }^{27}$ De la même manière que la revendication de l'appartenance de Dokdo/Takeshima à la Corée du Sud est une question de souveraineté 
nationale, alors la déclaration du Premier Ministre japonais que le kimchi et yakiniku ( bulgogi en coréen) sont deux plats représentatifs de la cuisine japonaise apparaît comme une violation de la souveraineté nationale.

A partir de 2002 et le «boom de la culture sud-coréenne » kanryū būmu 韓流ブームau Japon, beaucoup d'actrices et acteurs, chanteuses et chanteurs sud-coréens devinrent très populaires dans l'archipel. Un certain nombre d'entre eux en vinrent même à participer à des émissions de divertissement japonais. En 2010, le Seoul Culture Today titrait un article «Kara, Kimuchi, une déclaration de traitre! A-t-elle perdu la raison?» “카라 기무치 매 국발언 ! 제정 신인가 ?". ${ }^{28}$ Lors d'une émission, les membres du girls-band Kara utilisèrent les mots japonais afin de parler de plats coréens. À la place de Bulgogi : Yakiniku; à la place de Gimbap : Norimaki. Bien que toutes les chanteuses de ce groupe soient critiquées dans cet article, le nom d'une seule chanteuse était citée, Han Seung-yeon. Cette dernière avait prononcé kimchi à la manière japonaise : ki-mu-chi. Déjà en 2009, l'acteur Jeong Useong avait créé un précédent lors d'un programme japonais durant lequel il avait écrit en alphabet romain kimuchi et non kimchi. Après cet incident, il dut présenter des excuses publiques.

Les liens entre le kimchi et le Japon apparaissent également dans la franchise des films « Marrying the Mafia » Gamun ui Yeonggoang 가문의 영 광. Déjà dans le troisième opus en 2006 de la franchise des films " Marrying the Mafia », l'intérêt des Japonais pour le kimchi se trouvait exprimé au détour d'un dialogue. Mais la différence entre kimchi et kimuchi apparaît dans le film « Marrying the Mafia 4 » (2011) :

홍회장 : 어째 이렇게 됐냐? 이게 뭇이여 ?

비서 : 아 저... 미주시장은 그런대로 버티고 있는데, 일본이 문젭니다.

홍회장 : 아 일본이 한류 땜시 그동안에 짭짤하게 솔찬히 재미봤는디. 왜 이 바닥 이여 ?

비서: 김치 매출이 줄어서 그렇습니다. 그 일본 식품 회사들이 저마다 기무치를 만들어서 시장에 내놓는 바람에...

홍회장 : 기무치 ?

비서 : 예. 우리 김치보다 들 맵고요. 달달한 맛을 넣어서 싸무라이들이 좋아하는 것 같습니다.

홍회장 : 아 기무치하고 김치하고 완전 다르제! 아, 기무치는 빽사리여 !

Directrice Hong: Comment ça se fait que cela soit comme ça? Qu'est ce que c'est que ça?

Secrétaire: Ah, ça... Le marché américain se maintient, mais c'est le Japon le problème.

Directrice Hong: Ah, c'était très lucratif au Japon au moment du hallyu. Pourquoi c'est aussi bas?

Secrétaire : Les exportations de kimchi ont diminué. C'est à cause des entreprises japonaises qui mettent sur le marché le kimuchi qu'ils fabriquent.

Directrice Hong : Kimuchi?

Secrétaire : Oui. Il est moins épicé que notre kimchi. Ils y mettent un goût sucré et apparemment les samouraïs aiment ça.

Directrice Hong: Le kimuchi et le kimchi, ça n'a rien à voir ! Le kimuchi ce n'est qu'une pâle imitation.

Lors d'une interview à la télévision japonaise qui prend place à la fin du film, la directrice Hong insiste sur la différence de prononciation entre kimchi et kimuchi signifiant que la différence de prononciation n'est pas qu'un problème linguistique mais que la différence est également inhérente au produit en lui-même, le kimuchi ne pouvant être par définition du kimchi, ni pouvoir prétendre en être. En outre, le choix d'utiliser le terme samouraï ici n'est en rien anodin. Le caractère épicé du kimchi symbolise la 
masculinité. ${ }^{29}$ Le fait que le samouraï, symboles du guerrier japonais, préfèrent un kimchi moins épicé, souligne le manque de virilité de l'homme japonais en comparaison avec l'homme sud-coréen.

\section{Conclusion}

L'ouverture de l'économie au milieu des années 1980 marqua l'entrée dans la mondialisation de la Corée du Sud. Toutefois, cette libéralisation qui s'accompagna d'une occidentalisation donna l'impression de la destruction des fondements de la société et des traditions sud-coréennes. Comme souvent dans ce cas, s'ensuivit en réaction une période de recherche des traditions. Et comme dans bien d'autres cas encore, il s'agit en partie d'une réinvention de celles-ci. ${ }^{30}$ De plus, la revalorisation de la gastronomie nationale s'inscrit dans un ensemble où la culture coréenne elle-même est redécouverte, les produits culturels et alimentaires étrangers étaient devenus plus nombreux dans le cadre de la mondialisation. Cette volonté de défense de la culture coréenne vint d'une partie de la population, mais le gouvernement sud-coréen, pour faire face à la libéralisation débutée au milieu des années 1980 et à la faiblesse économique de ses industries culturelles et autres, décida de faire sienne la mondialisation. Les moyens de soutenir les entreprises et de consolider l'identité nationale se concrétisèrent par l'organisation de festivals concernant tous les secteurs culturels (gastronomie, cinéma, etc.). La nourriture, en dépassant le cadre de réponse à des besoins biologiques, acquiert le statut d'apports à la construction de l'identité culturelle. Toutefois, la mondialisation de la gastronomie sud-coréenne ne signifie pas son adaptation aux goût des étrangers, mais bien plutôt la révélation au monde entier de ce qu'est la cuisine coréenne " authentique », comme nous venons de le voir dans les deux films "Sikgaek» et "Sikgaek 2 ». Asakura Toshio fait remarquer que le kimchi a été choisi pour figurer dans le titre d'un livre rassemblant des contributions sur le thème de l'économie dans la mondialisation "The Kimchi Matters ". ${ }^{31}$ Il faut y ajouter le livre écrit par Choe Seok-yeong «Le patriotisme du Kimchi » Gimchi egukchuui김치 애국주의. Cet ouvrage analyse l'image du Japon diffusée par les médias sud-coréens dans différents domaines, du football aux problèmes liés à l'histoire en passant par la consommation de viande de baleine. Bien qu'il ne s'agisse pas des seuls livres à comporter le mot kimchi dans leur titre, nous pensons toutefois qu'ils nous éclairent quant aux deux principales tendances du nationalisme sud-coréen actuel que nous avons essayé de dégager tout au long de cet article.

Nous avons vu dans les deux films, mais également dans d'autres productions du même genre, que le Japon était l'adversaire en matière de kimuchi mais également un adversaire historique. Si des différences sont pointées du doigt concernant le processus de fabrication du kimchi, nous pensons que, au moins autant que l'authenticité, l'intérêt économique est un facteur important dans la défense de cet aliment. L'augmentation des tonnes de kimchi produites et exportées par le Japon représentant une spoliation des gains potentiels des entreprises sud-coréennes. De plus, la défense d'un plat reconnu aujourd'hui comme national en Corée du Sud est également un moyen pour le gouvernement sud-coréen de défendre le concept d'identité nationale. La nation apparaît dans le cas de la Corée du Sud comme moyen pour l'État de se réaffirmer dans la mondialisation, mais également de soutenir les entreprises du pays, publiques ou privées. La preuve de cette revalorisation du "national » est le terme " produit en Corée du Sud » 한국산, devenu un gage de qualité $e^{32}$ ne se limitant pas uniquement aux aliments, mais 
s'étendant aux vêtements et aux produits électroniques de grandes marques sudcoréennes mondialement connues et utilisées. Le kimchi étant désormais reconnu par l'UNESCO comme appartenant à la gastronomie coréenne, l'avenir dira s'il continue ou cesse d'être associé à l'Autre japonais dans l'imaginaire cinématographique.

\section{NOTES}

1. Maria Osetrova, "Korean Cuisine and its Role in the Process of National Identity Making in Contemporary South Korea", Korean Studies in Shift, Proceedings of the 2010 Pacific Asian Conference on Korean Studies, 2010, p.180.

2. Gi-wook Shin, Ethnic Nationalism in Korea: Genealogy, Politics, and Legacy, Stanford University Press, 2006, p.216.

3. Maria Osetrova, op. cit., p.171. Kyung-Koo Han, "Some Food Are Good to Think", Korean Social Science Journal, vol. XXVII, ${ }^{\circ} 1,2000$, p.222.

4. Ibid., p.229.

5. On retrouve notamment une scène dans le film My Way (2011) où un lieutenant sadique de l'armée impériale japonaise s'étonne que seulement un petit groupe de Coréens aient pu survivre après une escarmouche. Il demande alors si les Chinois les ont distingués des Japonais à cause de l'odeur de kimchi.

6. Kyung-Koo Han, op. cit., p.231.

7. Kyung-Koo Han, op. cit., p.224.

8. Calvins Sims, "Cabbage Is Cabbage? Not to Kimchi Lovers; Koreans Take Issue With a Rendition of Their National Dish Made in Japan", http://www.nytimes.com/2000/02/05/business/cabbagecabbage-not-kimchi-lovers-koreans-take-issue-with-rendition-their.html (consulté le 24 juillet 2010).

9. Codex Alimentarius Commission, "Codex Standard For Kimchi", www.codexalimentarius.org\% 2Fdownload\%2Fstandards\%2F365\%

2FCXS_223e.pdf\&ei=ZqgyVMbnG8Xe7AbE6oG4Dg\&usg=AFQjCNG38BV9_kWud8TWgb3MWKEX6tOBg\&sig2=NFD9GPwEXx6RKh1j8504w\& Consulté le 12, mai, 2009.

10. Hong Byeong-gi 홍병기, Gimchi ijen gukje eumsik kimuchi nulreo suchul uui '김치' 이젠 국 제음식... '기무치' 눌러 수출우위 [Le kimchi, un mets désormais international... Des exportations supérieures à celles du kimuchi], http://article.joins.com/news/article/article.asp? total_id=955278 (consulté le 28 mars 2011).

11. Kyung-Koo Han, op. cit., p.222.

12. «La fin de l'automne correspond à la saison du kimjang, moment où les communautés préparent collectivement de grandes quantités de kimchi et les partagent de façon à ce que chaque foyer ait suffisamment de nourriture pour affronter le long et rigoureux hiver. Les ménagères suivent les prévisions météorologiques afin de déterminer à quelle date la température sera optimale pour préparer le kimchi. Le processus d'échange de kimchi entre les familles est l'occasion de partager des techniques innovantes et de nouvelles idées. Il existe des différences régionales et, les méthodes comme les ingrédients spécifiques employés pour le kimjang, sont considérés comme un héritage familial important, transmis le plus souvent par les belles-mères aux belles-filles nouvellement mariées. " "Le kimjang, préparation et partage du 
kimchi en République de Corée", http://www.unesco.org/culture/ich/index.php? $\mathrm{lg}=\mathrm{fr} \& \mathrm{pg}=00011 \& \mathrm{RL}=00881$ (Consulté le 22 décembre 2013).

13. Gi-Wook Shin, "The Paradox of Korean Globalization", http://iis-db.stanford.edu/ pubs/20125/Shin.pdf (consulté le 28 janvier 2009).

14. Hansik segyehwa ran 한식세 계화란 [Qu'est-ce que la mondialisation de la cuisine coréenne? ], http://www.hansik.org/kr/article.do?cmd=html (consulté le 09 février 2011).

15. En 1993, la part du cinéma sud-coréen dans le marché national était de $15,3 \%$. Un quota pour les films étrangers fut mis en place dès les années 1960. Malgré cela, le cinéma sud-coréen fut dépendant des importations de films étrangers afin de financer ses productions nationales asphyxiées par les règlementations durant les différentes dictatures militaires. Cependant, la durée réservée pour les films sud-coréens baissa. En 1993, une grande partie des directeurs de salles de cinéma ne respectait pas les quotas imposés, et il est estimé que seulement 48 jours sur les 146 réglementaires étaient réservés aux films sud-coréens.

16. Jong-hwa Jeong 정종화, Hanguk yeonghwasa 한국영화사 [Histoire du cinéma sud-coréen], Séoul, Hanguk yoeongsang Jaryoweon, 2008, p.238. Cette reconquête du marché peut paraître surprenante, elle se fit par les blockbusters à la sud-coréenne, superproductions produites avec des budgets gigantesques pour l'économie sud-coréenne (mais restant incomparables avec ceux d'Hollywood). Toutefois, cette réappropriation du marché national par des films sud-coréens n'est pas sans poser de problème quant aux possibilités de production dans la structure actuelle à cause de la situation de monopole de quelques chaebol. Paradoxalement, cela neutralise la diversité qu'est censé assurer le système de quota. Hyujong Kim김휴종, "Hanguk yeonghwa saneop goa dokdoajeom isyu" 한국영화산업과 독과점 이슈 [L'industrie cinématographique sudcoréenne et les problèmes de monopole], Munwha gyeongje yeongu, vol.7, n², 2004, p.64.

17. Okpyo MOON, "Dining Elegance and Authenticity: Archeology of Royal Court Cuisine in Korea", Korea Journal, Spring 2010, p.51.

18. Ibid., p.51.

19. Statistiques disponibles sur le site du Korean Film Council, http://www.kobis.or.kr/ (consulté le 14 mai 2010).

20. Le premier opus fut projeté sur 406 écrans et le second sur 357. Le nombre d'écrans total en Corée du Sud était de 1975 en 2007 et de 2004 en 2008.

21. Hyeong Min- $u$ 형 민우, Baeu kim jeongeun gwangju gimchi chukje honbo naseonda 배우 김정 은 ‘광주김치 축제’ 홍보 나선다 [L'actrice Kim Jeong-eun ambassadrice du 'Festival de Kimchi de Gwangju'] http://www.yonhapnews.co.kr/ bulletin/2009/07/28/0200000000AKR20090728097100054.HTML (consulté le 29 août 2009).

22. Cho Jae-eun, "Modern Mothers Lose in Reactionary 'Kimchi War'", http:// koreajoongangdaily.joins.com/news/article/article.aspx?aid=2918938. (consulté le 05 mai 2010).

23. Claude Fischler, "Food, Self and Identity", Social Science Information, n²7, 1988, p.275.

24. Ibid., p.278.

25. Kimura Kan木村韓, Kindai kankoku no nashonarizumu 近代韓国のナショナリズム [Le nationalisme sud-coréen moderne], Kyōto, Nakanishiya Shuppan, 2009, p.289.

26. Kozakai Toshiaki 小坂井敏晶, Minzoku to iu kyokō 民族という虚構 [La fiction appelée ethnie], Tōkyō, Chikumashobō, 2011, p.40.

27. Pour plus d'informations sur Dokdo/Takeshima, voir Philippe Pelletier, « Tumulte des flots entre Japon et Corée. À propos de la dénomination de la " mer du Japon », Annales de Géographie, t. 109, n613, 2000, pp. 279-305.

28. Wang Su-min 왕수민, Kara kimuchi maegukbareon jejeongshin inga카라 '기무치' 매국발언! 제정신인가? [Le «Kimuchi» de Kara, une expression de collabo! Est-elle saine d'esprit?], http://www.sctoday.co.kr/news/articleView.html?idxno=8304 (consulté le 04 avril 2011).

29. Kyung-Koo, op. cit., p.227. 
30. Kwang Ok Kim, "Rice Cuisine and Cultural Practice in Contemporary Korean Dietary Life", Korea Journal, vol. 51, $\mathrm{n}^{\circ} 1$, Spring 2010, p.32.

31. Asakura Toshio 朝倉敏夫, Gendai imin no tayōsei : ekkyō suru kimuchi 現代移民の多様性 : 越境するキムチ[La diversité de l'immigration contemporaine: le kimuchi qui dépasse les frontières], Kokuritsu Minzokugaku Hakubutsukan Chōsahōkoku, nº3 , 2009, p.66.

32. Kwang Ok Kim, op. cit., p.32.

\section{RÉSUMÉS}

Cet article analyse comment l'opposition entre le Japon et le patrimoine gastronomique de la Corée du Sud est utilisée dans la construction de l'identité nationale au travers des deux superproductions « Sikgaek » (2007) et «Sikgaek 2 » (2010). Les blockbusters produits localement depuis la fin des années 1990 sont devenus un pan incontournable de l'histoire de la culture populaire sud-coréenne et sont un vecteur spectaculaire du nationalisme sud-coréen lorsqu'ils traitent de l'histoire passée ou récente. 